Abdelfattah El Achab (Marrakech)

\title{
NEW WEIERSTRASS ELLIPTIC WAVE SOLUTIONS OF THE DAVEY-STEWARTSON EQUATION WITH POWER LAW NONLINEARITY
}

Abstract. Travelling wave solutions for the $(2+1)$-dimensional DaveyStewartson-type equations are studied by using the Weierstrass elliptic function method. As a result, some previously known solutions are recovered, and at the same time some new ones are also given including integrable ones. Moreover, three-dimensional and two-dimensional graphics of some solutions are plotted.

1. Introduction. The Davey-Stewartson (DS) equations for the function $q=q(x, y, t)$ are (see 7$]$ )

$$
\begin{aligned}
& i q_{t}+a q_{x x}+q_{y y}+b|q|^{2} q-2 q r_{x}=0, \\
& r_{x x}-a r_{y y}-b\left(|q|^{2}\right)_{x x}=0,
\end{aligned}
$$

where $q$ is the (complex valued) envelope of the wave packet associated with the fast oscillations, and $r$ is the induced mean flow, $x$ and $y$ are the slow, horizontal scales parallel and perpendicular to the fast oscillations, respectively, whereas $t$ is the slow time in the group velocity frame. As usual, irrotational flow of an inviscid fluid is studied, and $q$ is connected with the velocity potential. Of particular significance is the parameter $a$, and the cases $a=1$ and $a=-1$ are called the DS I and DS II equations, respectively. The constant $b$ measures the cubic nonlinearity. The DS equation is a two-dimensional generalization of the nonlinear Schrödinger (NLS) equation. Much work has been done in the past few decades to study this equation. Especially, in 1988, Boiti et al. [5] considered the DS equation and constructed solutions that

2020 Mathematics Subject Classification: 35Q53, 35B, 35Q51, 37K10.

Key words and phrases: travelling wave solutions, exact solutions, Weierstrass elliptic function method, Davey-Stewartson-type equations.

Received 23 January 2019.

Published online 31 August 2020. 
decay exponentially in all directions. Using the inverse scattering method, Fokas and Santini [14, 15] derived such solutions and named them dromions. Hietarinta and Hirota [17, 18] and Radha and Lakshmanan [27] found multidromion solutions or one-dromion solutions to the DS equation by the bilinear method. Their works developed the study of the DS equation in an interesting direction. Recently, many new methods to construct exact solutions of the DS equation have been established and developed, such as the sine-cosine method [3, 32], extended Weierstrass transformation method [16], trial equation method and ansatz approach [25], extended Jacobian elliptic function expansion method [4], homotopy analysis method [21], homotopy perturbation method [33], first integral method [22], exp-function method, $\left(\frac{G^{\prime}}{G}\right)$ method [8], uniform algebraic method [13] etc. The solutions of the DS equation have been applied in plasma physics, nonlinear optics, hydrodynamics, etc. For example, the solutions of the DS equation can describe the interaction between a spatiotemporal optical pulse and adequately matched microwaves 23.

The purpose of this paper is to apply the Weierstrass elliptic function method $912,26,28]$ to the following $(2+1)$-dimensional Davey-Stewartson-type equations with power law nonlinearity [8, 29]:

$$
\begin{aligned}
& i q_{t}+a\left(q_{x x}+q_{y y}\right)+b|q|^{2 n} q-\alpha q r=0, \\
& r_{x x}+r_{y y}+\beta\left(|q|^{2 n}\right)_{x x}=0 .
\end{aligned}
$$

Here, $q$ and $r$ are the dependent variables while $x, y$ and $t$ are the independent variables. The first two independent variables are the spatial variables while $t$ represents time. The exponent $n$ is the power law parameter. It is necessary to have $n>0$. In (1.2), $q$ is a complex valued function while $r$ is a real valued function. Also, $a, b, \alpha$, and $\beta$ are all constant coefficients. The authors of 8 and [29] obtained some exact explicit solutions of 1.2 .

The objective of this paper is to investigate the travelling wave solutions of (1.2) systematically, by applying the Weierstrass elliptic function method. It will be shown that some previously known solutions are recovered, and some new ones are found.

The paper is organized as follows. In Section 2, we outline the Weierstrass elliptic function method. In Section 3, we give some particular travelling wave solutions of 1.2 . Finally, some corollaries are given in Section 4. In the Appendix, we give some elementary features and properties of Weierstrass functions.

2. Weierstrass elliptic functions. When searching for solutions of some evolutionary equations, we encounter the following ordinary differential equation [26, 31]: 


$$
\left(\frac{d \varphi(\xi)}{d \xi}\right)^{2}=a_{0} \varphi^{4}+4 a_{1} \varphi^{3}+6 a_{2} \varphi^{2}+4 a_{3} \varphi+a_{4}=P(\varphi) .
$$

In [31], Whittaker and Watson introduced two invariants:

$$
\begin{aligned}
& g_{2}=a_{0} a_{4}-4 a_{1} a_{3}+3 a_{2}^{2}, \\
& g_{3}=a_{0} a_{2} a_{4}+2 a_{1} a_{2} a_{3}-a_{2}^{3}-a_{0} a_{3}^{2}-a_{1}^{2} a_{4},
\end{aligned}
$$

and the discriminant $\Delta=g_{2}^{3}-27 g_{3}^{2}$. Then solutions $\varphi$ of 2.1 can be expressed in terms of elliptic functions $\wp 30,31$ :

$$
\varphi(\xi)=\varphi_{0}+\frac{P^{\prime}\left(\varphi_{0}\right)}{4\left[\wp\left(\xi, g_{2}, g_{3}\right)-\frac{1}{24} P^{\prime \prime}\left(\varphi_{0}\right)\right]},
$$

where $\varphi_{0}$ is one of the roots of the polynomial $P(\varphi)$, and $P^{\prime}\left(\varphi_{0}\right), P^{\prime \prime}\left(\varphi_{0}\right)$ respectively denote the first and second derivative of $P(\varphi)$ with respect to $\varphi$ at $\varphi_{0}$. In particular, if $\Delta=g_{2}^{3}-27 g_{3}^{2}=0$, then the Weierstrass elliptic function $\wp\left(\xi, g_{2}, g_{3}\right)$ satisfies these conditions [1]:

$$
\begin{array}{lll}
\wp\left(\xi, 12 s^{2}, 8 s^{3}\right)=-s+3 s \sin ^{-2}(\sqrt{3 s} \xi), & g_{2}>0, & g_{3}>0, \\
\wp\left(\xi, 12 s^{2},-8 s^{3}\right)=s+3 s \sinh ^{-2}(\sqrt{3 s} \xi), & g_{2}>0, & g_{3}<0,
\end{array}
$$

where $s=\frac{1}{2} \sqrt[3]{\left|g_{3}\right|}$. Once we get a solution of 2.1 of the form 2.4 , we will get exact expressions of solutions of many partial differential equations. Suppose that $\Delta=g_{2}^{3}-27 g_{3}^{2}=0$. It is obvious that if $g_{2}>0, g_{3}>0$, then there exist periodic solutions of the original evolutionary equation:

$$
\varphi(\xi)=\varphi_{0}+\frac{P^{\prime}\left(\varphi_{0}\right)}{4\left[-\frac{e_{1}}{2}-\frac{P^{\prime \prime}\left(\varphi_{0}\right)}{24}+\frac{3}{2} e_{1} \csc ^{2}\left(\sqrt{\frac{3}{2} e_{1}} t\right)\right]}, \quad \Delta=0, g_{3}>0,
$$

while if $g_{2}>0, g_{3}<0$, then there exist solitary solutions:

$$
\varphi(\xi)=\varphi_{0}+\frac{P^{\prime}\left(\varphi_{0}\right)}{4\left[e_{1}-\frac{P^{\prime \prime}\left(\varphi_{0}\right)}{24}+3 e_{1} \operatorname{csch}^{2}\left(\sqrt{3 e_{1}} t\right)\right]}, \quad \Delta=0, g_{3}<0,
$$

where $e_{1}=\sqrt[3]{\left|g_{3}\right|}$ in 2.6. and $e_{1}=\frac{1}{2} \sqrt[3]{\left|g_{3}\right|}$ in 2.7.

3. The 2D Davey-Stewartson equation with power law nonlinearity. We assume that a travelling wave solution of $(1.2)$ is of the form

$$
\begin{array}{r}
q(x, y, t)=\exp (i \theta) g(\xi), \quad r(x, y, t)=h(\xi), \\
\xi=k x+\lambda y-\nu t, \quad \theta=p x+s y+\omega t
\end{array}
$$

where $k, \lambda, \nu, p, s$ and $\omega$ are real constants. Then 1.2 becomes the system of ODEs 


$$
\begin{aligned}
& (-\nu+2 a(p k+s \lambda)) g^{\prime}(\xi)=0, \\
& a\left(k^{2}+\lambda^{2}\right) g^{\prime \prime}-\left(\omega+a\left(p^{2}+s^{2}\right)\right) g+b g^{2 n+1}-\alpha g h=0, \\
& \left(k^{2}+\lambda^{2}\right) h^{\prime \prime}+\beta k^{2}\left(g^{2 n}\right)^{\prime \prime}=0 .
\end{aligned}
$$

where the primes denote derivatives with respect to $\xi$. Equation (3.4) can then be integrated term by term two times where the integration constants are taken to be zero. This converts it into

$$
h=-\frac{\beta k^{2}}{k^{2}+\lambda^{2}} g^{2 n} .
$$

Substituting (3.5) into 3.3, we have

$$
a\left(k^{2}+\lambda^{2}\right) g^{\prime \prime}-\left(\omega+a\left(p^{2}+s^{2}\right)\right) g+\left(b+\frac{\alpha \beta k^{2}}{k^{2}+\lambda^{2}}\right) g^{2 n+1}=0 .
$$

By multiplying $(3.6)$ by $g^{\prime}$ and integrating once again, we get

$$
\left(g^{\prime}\right)^{2}=K+\delta g^{2}-\frac{\gamma}{n+1} g^{2 n+2}
$$

where $\delta=\frac{\omega+a\left(p^{2}+s^{2}\right)}{a\left(k^{2}+\lambda^{2}\right)}, \gamma=\frac{1}{a\left(k^{2}+\lambda^{2}\right)}\left(b+\frac{\alpha \beta k^{2}}{k^{2}+\lambda^{2}}\right)$ and $K$ is the integration constant. The transformation $g=\varphi^{p}, p \neq 0,1$, turns the above equation to

$$
\left(\frac{d \varphi}{d \xi}\right)^{2}=\frac{1}{p^{2}}\left[K \varphi^{2-2 p}+\delta \varphi^{2}-\frac{\gamma}{n+1} \varphi^{2 n p+2}\right] .
$$

If we want to guarantee the integrability of (3.8), the powers of $\phi$ have to be integers between 0 and 4 [19], and therefore we have the following possible cases:

- If $K=0$, then $p \in\left\{-\frac{1}{n},-\frac{1}{2 n}, \frac{1}{2 n}, \frac{1}{n}\right\}$.

In the following it will be proved that $p=\frac{1}{2 n}$ and $p=\frac{1}{n}$ give the same solution of 2.1), and therefore we only consider $p=\frac{1}{2 n}$. For the same reason, for $p=-\frac{1}{2 n}$ and $p=-\frac{1}{n}$ we will only consider $p=-\frac{1}{2 n}$.

- If $K \neq 0$, then $n=2$ and $p= \pm \frac{1}{2}$.

Next, by using the results obtained in the preceding sections, we will construct the corresponding solutions of $(3.8)$ in the above cases.

\subsection{Case 1}

Case 1(i): $K=0, p=\frac{1}{2 n}$. In this case, 3.8 takes the form

$$
\left(\frac{d \varphi}{d \xi}\right)^{2}=4 n^{2}\left[\delta \varphi^{2}-\frac{\gamma}{n+1} \varphi^{3}\right]=P(\varphi) .
$$

The polynomial $P(\varphi)$ has two roots: $\varphi_{0}=0$ (with multiplicity two) and $\varphi_{0}=\delta(n+1) / \gamma$. From (2.4), for each root $\varphi_{0}$ a solution of (3.9) can be 
found to be

$$
\varphi=\frac{3 \varphi_{0} \wp\left(\xi, g_{2}, g_{3}\right)+5 n^{2} \delta \varphi_{0}-\frac{6 n^{2} \gamma}{n+1} \varphi_{0}^{2}}{3 \wp\left(\xi, g_{2}, g_{3}\right)-n^{2} \delta+\frac{3 n^{2} \gamma}{n+1} \varphi_{0}}
$$

where the invariants are

$$
g_{2}=48 n^{4} \delta^{2}, \quad g_{3}=-64 n^{6} \delta^{3} .
$$

The root $\varphi_{0}=0$ gives the trivial solution $\varphi=0$, and the nonzero solution of $P(\varphi)=0$ can be easily found. By substituting $\varphi_{0}=\delta(n+1) / \gamma$ in (3.10), we get

$$
\varphi=\frac{\delta(n+1)}{\gamma} \frac{3 \wp\left(\xi, g_{2}, g_{3}\right)-n^{2} \delta}{3 \wp\left(\xi, g_{2}, g_{3}\right)+2 n^{2} \delta} .
$$

Since $\Delta=0$, we find a periodic wave solution to 3.9 from 2.6 :

$$
\varphi=\frac{\delta(n+1)}{\gamma} \sec ^{2}(n \sqrt{-\delta} \xi)
$$

for $\delta<0$. A solitary wave solution of 3.9 is obtained from 2.7 :

$$
\varphi=\frac{\delta(n+1)}{\gamma} \operatorname{sech}^{2}(n \sqrt{\delta} \xi)
$$

for $\delta>0$. Changing to the original variables, the periodic wave solution of 1.2 (for $\delta<0$ ) and the solitary wave solution (for $\delta>0$ ) can be written respectively as

$$
\begin{aligned}
& q(x, t)=\exp (i \theta)(\sqrt{\delta(n+1) / \gamma} \sec (n \sqrt{-\delta} \xi))^{1 / n} \\
& r(x, t)=-\frac{\beta k^{2}}{k^{2}+\lambda^{2}} \frac{\delta(n+1)}{\gamma}(\sec (n \sqrt{-\delta} \xi))^{2}
\end{aligned}
$$

and

$$
\begin{aligned}
& q(x, t)=\exp (i \theta)(\sqrt{\delta(n+1) / \gamma} \operatorname{sech}(n \sqrt{\delta} \xi))^{1 / n}, \\
& r(x, t)=-\frac{\beta k^{2}}{k^{2}+\lambda^{2}} \frac{\delta(n+1)}{\gamma}(\operatorname{sech}(n \sqrt{\delta} \xi))^{2} .
\end{aligned}
$$

We will now show that by choosing $p=\frac{1}{n}$ in 3.8 instead $p=\frac{1}{2 n}$, we will recover the same solutions for 1.2 .

In this case, (3.8) becomes

$$
\left(\frac{d \varphi}{d \xi}\right)^{2}=4 n^{2}\left[\delta \varphi^{2}-\frac{\gamma}{n+1} \varphi^{4}\right]=P(\varphi) .
$$

The fourth order polynomial $P(\varphi)$ has two roots: $\varphi_{0}=0$ (double) and $\varphi_{0}=$ $\pm \sqrt{\delta(n+1) / \gamma}$. From 2.4, the solution of 3.17) in terms of $\varphi_{0}$ is

$$
\varphi=\frac{3 \varphi_{0} \wp\left(\xi, g_{2}, g_{3}\right)+5 n^{2} \delta \varphi_{0}-\frac{6 n^{2} \gamma}{n+1} \varphi_{0}^{3}}{3 \wp\left(\xi, g_{2}, g_{3}\right)-n^{2} \delta+\frac{6 n^{2} \gamma}{n+1} \varphi_{0}^{2}}
$$


where the invariants are

$$
g_{2}=\frac{4}{3} n^{4} \delta^{2}, \quad g_{3}=-\frac{8}{27} n^{6} \delta^{3} .
$$

Then for $\varphi_{0}= \pm \sqrt{\delta(n+1) / \gamma}$, a nontrivial solution of $(3.17)$ is

$$
\varphi= \pm \sqrt{\frac{\delta(n+1)}{\gamma}} \frac{3 \wp\left(\xi, g_{2}, g_{3}\right)-n^{2} \delta}{3 \wp\left(\xi, g_{2}, g_{3}\right)+5 n^{2} \gamma} .
$$

Since $\Delta=0$, it is easy to see from (2.6), 2.7) that the solutions (3.20) give rise to the same periodic wave and solitary wave solutions of 1.2 as in (3.15), 3.16.

CASE 1(ii): $K=0, p=\frac{-1}{2 n}$. In this case, 3.8 takes the form

$$
\left(\frac{d \varphi}{d \xi}\right)^{2}=4 n^{2}\left[\delta \varphi^{2}-\frac{\gamma}{n+1} \varphi\right]=P(\varphi) .
$$

The second order polynomial $P(\varphi)$ has two roots: $\varphi_{0}=0$ and $\varphi_{0}=\frac{\gamma}{\delta(n+1)}$. The solution of (3.21), obtained from 2.4, is

$$
\varphi=\frac{3 \varphi_{0} \wp\left(\xi, g_{2}, g_{3}\right)+5 n^{2} \delta \varphi_{0}-\frac{3 n^{2} \gamma}{n+1}}{3 \wp\left(\xi, g_{2}, g_{3}\right)-n^{2} \gamma}
$$

where the invariants are given by (3.11). Substituting $\varphi_{0}=0$ into 3.22), we get

$$
\varphi=-\frac{3 n^{2} \gamma}{(n+1)\left(3 \wp\left(\xi, g_{2}, g_{3}\right)-n^{2} \delta\right)} .
$$

Since $\Delta=0$, from $(2.6)$ and $(2.7)$, a periodic wave solution and a solitary wave solution of (3.21) can be written respectively as

$$
\varphi=\frac{\gamma}{\delta(n+1)} \sin ^{2}(n \sqrt{-\delta} \xi) \quad(\delta<0)
$$

and

$$
\varphi=-\frac{\gamma}{\delta(n+1)} \sinh ^{2}(n \sqrt{\delta} \xi) \quad(\delta>0)
$$

Therefore, when $\delta<0,1.2$ has the following periodic wave solution:

$$
\begin{array}{r}
q(x, t)=\exp (i \theta)(\sqrt{\delta(n+1) / \gamma} \csc (n \sqrt{-\delta} \xi))^{1 / n}, \\
r(x, t)=-\frac{\beta k^{2}}{k^{2}+\lambda^{2}} \frac{\delta(n+1)}{\gamma}(\csc (n \sqrt{-\delta} \xi))^{2}
\end{array}
$$

when $\delta>0,1.2$ has the following solitary wave solution:

$$
\begin{aligned}
& q(x, t)=\exp (i \theta)(\sqrt{-\delta(n+1) / \gamma} \operatorname{csch}(n \sqrt{\delta} \xi))^{1 / n}, \\
& r(x, t)=\frac{\beta k^{2}}{k^{2}+\lambda^{2}} \frac{\delta(n+1)}{\gamma}(\operatorname{csch}(n \sqrt{\delta} \xi))^{2} .
\end{aligned}
$$


On substituting the second root $\varphi_{0}=\frac{\gamma}{\delta(n+1)}$ into 3.22 , we get

$$
\varphi=\frac{\gamma}{\delta(n+1)} \frac{3 \wp\left(\xi, g_{2}, g_{3}\right)+2 n^{2} \delta}{3 \wp\left(\xi, g_{2}, g_{3}\right)-n^{2} \delta} .
$$

So from 2.6 and (2.7), the solution can be expressed as

$$
\varphi=\frac{\gamma}{\delta(n+1)} \cos ^{2}(n \sqrt{-\delta} \xi) \quad(\delta<0)
$$

and

$$
\varphi=\frac{\gamma}{\delta(n+1)} \cosh ^{2}(n \sqrt{\delta} \xi) \quad(\delta>0) .
$$

Thus, we get the solutions of $(1.2)$ which were given by $(3.15)$ and $(3.16)$. In addition, it can be proved that the choice $p=-\frac{1}{n}$ in 3.8 gives exactly the same solutions for 1.2.

Exact traveling wave solutions of the $(2+1)$-dimensional Davey-Stewartson equation with power law nonlinearity can be obtained by using the above results:

TheOREM 1. The $(2+1)$-dimensional Davey-Stewartson equation with power law nonlinearity has solutions described as follows:

(1) When $\delta<0$, there exist the following explicit periodic wave solutions:

$$
\begin{aligned}
& q_{1}(x, y, t)=\exp (i \theta)(\sqrt{\delta(n+1) / \gamma} \sec (n \sqrt{-\delta} \xi))^{1 / n} \\
& r_{1}(x, y, t)=-\frac{\beta k^{2}}{k^{2}+\lambda^{2}} \frac{\delta(n+1)}{\gamma}(\sec (n \sqrt{-\delta} \xi))^{2}
\end{aligned}
$$

and also

$$
\begin{aligned}
& q_{2}(x, y, t)=\exp (i \theta)(\sqrt{\delta(n+1) / \gamma} \csc (n \sqrt{-\delta} \xi))^{1 / n} \\
& r_{2}(x, y, t)=-\frac{\beta k^{2}}{k^{2}+\lambda^{2}} \frac{\delta(n+1)}{\gamma}(\csc (n \sqrt{-\delta} \xi))^{2}
\end{aligned}
$$

(2) When $\delta>0$, there exist the following explicit solitary wave solutions:

$$
\begin{aligned}
& q_{3}(x, y, t)=\exp (i \theta)(\sqrt{\delta(n+1) / \gamma} \operatorname{sech}(n \sqrt{\delta} \xi))^{1 / n} \\
& r_{3}(x, y, t)=-\frac{\beta k^{2}}{k^{2}+\lambda^{2}} \frac{\delta(n+1)}{\gamma}(\operatorname{sech}(n \sqrt{\delta} \xi))^{2}
\end{aligned}
$$

and also

$$
\begin{aligned}
& q_{4}(x, y, t)=\exp (i \theta)(\sqrt{-\delta(n+1) / \gamma} \operatorname{csch}(n \sqrt{\delta} \xi))^{1 / n}, \\
& r_{4}(x, y, t)=\frac{\beta k^{2}}{k^{2}+\lambda^{2}} \frac{\delta(n+1)}{\gamma}(\operatorname{csch}(n \sqrt{\delta} \xi))^{2} .
\end{aligned}
$$

REMARK 3.1. 1. When $\delta<0$, the solutions (3.31) that we obtained coincide with the solutions (3.9) which were obtained by the dynamical system 
method by J. Shi et al. [29], When $\delta>0$, the solutions (3.33)-(3.34) we obtained coincide with those obtained by J. Shi et al. [29, (3.7)-(3.1)].

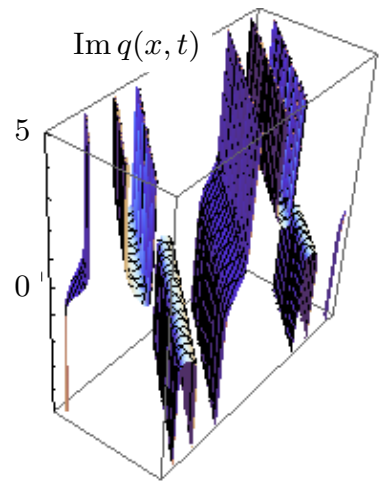

(a)
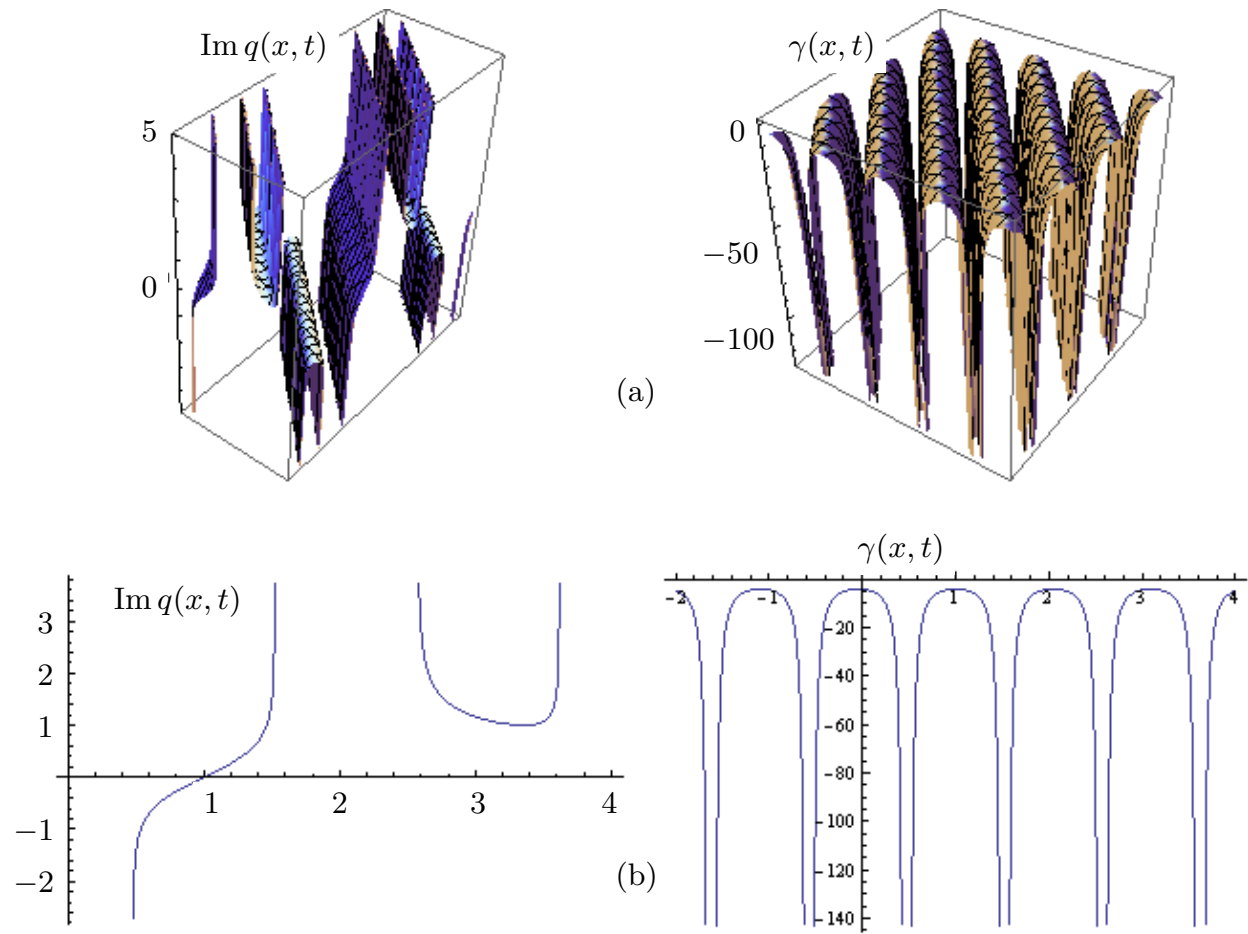

Fig. 1. (a) $3 \mathrm{D}$ surfaces of 3.31. (b) $2 \mathrm{D}$ surfaces of 3.31 for $n=3, \delta=\gamma=-1$, $k=p=v=-\omega=1, y=0$.

\subsection{Case 2}

CASE $2(\mathrm{i}): K \neq 0, n=2, p=\frac{1}{2}$. In this case, (3.8) takes the form

$$
\left(\frac{d \varphi}{d \xi}\right)^{2}=4\left[\delta \varphi^{2}-\frac{\gamma}{3} \varphi^{4}+K \varphi\right]=P(\varphi) .
$$

Now, solutions of (3.35) can be deduced from (2.4):

$$
\varphi=\frac{3 \varphi_{0} \wp\left(\xi, g_{2}, g_{3}\right)+5 \delta \varphi_{0}-2 \gamma \varphi_{0}^{3}+3 K}{3 \wp\left(\xi, g_{2}, g_{3}\right)-\delta+2 \gamma \varphi_{0}^{2}}
$$

where the invariants are given by

$$
g_{2}=4 \delta^{2}, \quad g_{3}=4\left(-\delta^{3}+9 \gamma K^{3}\right) / 27 .
$$


So we can obtain the general expressions for solutions to 1.2 :

$$
\begin{aligned}
& q(x, y, t)=\exp (i \theta)\left(\frac{3 \varphi_{0} \wp\left(\xi, g_{2}, g_{3}\right)+5 \delta \varphi_{0}-2 \gamma \varphi_{0}^{3}+3 K}{3 \wp\left(\xi, g_{2}, g_{3}\right)-\delta+2 \gamma \varphi_{0}^{2}}\right)^{1 / 2}, \\
& r(x, y, t)=-\frac{\beta k^{2}}{k^{2}+\lambda^{2}}\left(\frac{3 \varphi_{0} \wp\left(\xi, g_{2}, g_{3}\right)+5 \delta \varphi_{0}-2 \gamma \varphi_{0}^{3}+3 K}{3 \wp\left(\xi, g_{2}, g_{3}\right)-\delta+2 \gamma \varphi_{0}^{2}}\right)^{2} .
\end{aligned}
$$
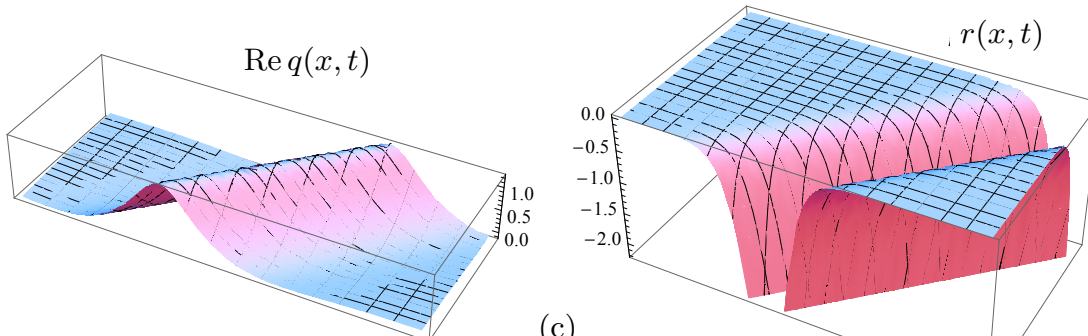

(c)

c)
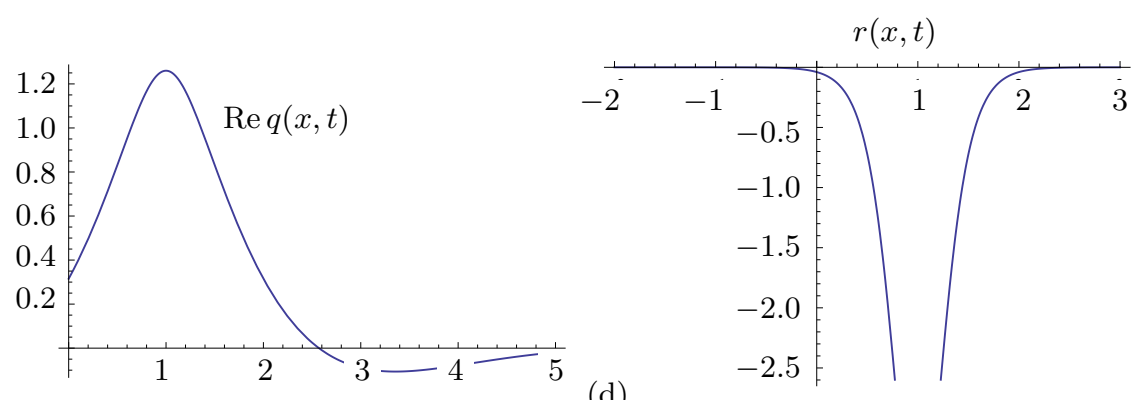

(d)

Fig. 2. (c) 3D surfaces of (3.33). (d) $2 \mathrm{D}$ surfaces of 3.33 for $n=3, \delta=\gamma=1, k=p=$ $v=-\omega=1, y=0$.

For example, taking the simplest root $\varphi_{0}=0$ of $P(\varphi)$ in (3.38), we obtain the solution of 1.2 given by

$$
\begin{aligned}
& q(x, y, t)=\exp (i \theta)\left(\frac{3 K}{3 \wp\left(\xi, g_{2}, g_{3}\right)-\delta}\right)^{1 / 2} \\
& r(x, y, t)=-\frac{\beta k^{2}}{k^{2}+\lambda^{2}}\left(\frac{3 K}{3 \wp\left(\xi, g_{2}, g_{3}\right)-\delta}\right)^{2} .
\end{aligned}
$$

CASE 2(ii): $K \neq 0, n=2, p=-\frac{1}{2}$. In this case, 3.8 has the form

$$
\left(\frac{d \varphi}{d \xi}\right)^{2}=4\left[\delta \varphi^{2}-\frac{\gamma}{3}+K \varphi^{3}\right]=P(\varphi)
$$


Using a similar procedure to Case 2(ii), we can get the following general expression for solutions to 1.2 :

$$
\begin{aligned}
& q(x, y, t)=\exp (i \theta)\left(\frac{3 \varphi_{0} \wp\left(\xi, g_{2}, g_{3}\right)+5 \delta \varphi_{0}-2 \gamma \varphi_{0}^{3}+3 K}{3 \wp\left(\xi, g_{2}, g_{3}\right)-\delta+2 \gamma \varphi_{0}^{2}}\right)^{-1 / 2}, \\
& r(x, y, t)=-\frac{\beta k^{2}}{k^{2}+\lambda^{2}}\left(\frac{3 \varphi_{0} \wp\left(\xi, g_{2}, g_{3}\right)+5 \delta \varphi_{0}-2 \gamma \varphi_{0}^{3}+3 K}{3 \wp\left(\xi, g_{2}, g_{3}\right)-\delta+2 \gamma \varphi_{0}^{2}}\right)^{-2} .
\end{aligned}
$$

SubCASE 1: $K<0, n=2, p=-\frac{1}{2}, \delta>0$ and $\gamma=0$. In this case the simple root of $P(\varphi)=0$ is $\varphi_{0}=-\delta / K$. Using (2.5) and inserting $\varphi_{0}$ into (2.4) yields

$$
\varphi(\xi)=\frac{-\delta}{K} \operatorname{sech}^{2}(\sqrt{\delta} \xi)
$$

So, 1.2 has the following explicit solitary wave solutions:

$$
\begin{aligned}
& q(x, y, t)=\exp (i \theta)\left(\frac{-\delta}{K} \operatorname{sech}^{2}(\sqrt{\delta} \xi)\right)^{1 / 2} \\
& r(x, y, t)=-\frac{\beta k^{2}}{k^{2}+\lambda^{2}}\left(\frac{-\delta}{K} \operatorname{sech}^{2}(\sqrt{\delta} \xi)\right)^{2} .
\end{aligned}
$$

The results of this subsection are summarized in the following proposition:

Proposition 3.1. (1) When $K \neq 0, n=2$, equation 1.2 has the following solutions:

$$
\begin{aligned}
& q(x, y, t)=\exp (i \theta)\left(\frac{3 \varphi_{0} \wp\left(\xi, g_{2}, g_{3}\right)+5 \delta \varphi_{0}-2 \gamma \varphi_{0}^{3}+3 K}{3 \wp\left(\xi, g_{2}, g_{3}\right)-\delta+2 \gamma \varphi_{0}^{2}}\right)^{1 / 2} \\
& r(x, y, t)=-\frac{\beta k^{2}}{k^{2}+\lambda^{2}}\left(\frac{3 \varphi_{0} \wp\left(\xi, g_{2}, g_{3}\right)+5 \delta \varphi_{0}-2 \gamma \varphi_{0}^{3}+3 K}{3 \wp\left(\xi, g_{2}, g_{3}\right)-\delta+2 \gamma \varphi_{0}^{2}}\right)^{2} .
\end{aligned}
$$

For example, for $\varphi_{0}=0$, a solution of (1.2) is given by

$$
\begin{aligned}
& q(x, y, t)=\exp (i \theta)\left(\frac{3 K}{3 \wp\left(\xi, g_{2}, g_{3}\right)-\delta}\right)^{1 / 2}, \\
& r(x, y, t)=-\frac{\beta k^{2}}{k^{2}+\lambda^{2}}\left(\frac{3 K}{3 \wp\left(\xi, g_{2}, g_{3}\right)-\delta}\right)^{2}
\end{aligned}
$$

and another one by

$$
q(x, y, t)=\exp (i \theta)\left(\frac{3 \varphi_{0} \wp\left(\xi, g_{2}, g_{3}\right)+5 \delta \varphi_{0}-2 \gamma \varphi_{0}^{3}+3 K}{3 \wp\left(\xi, g_{2}, g_{3}\right)-\delta+2 \gamma \varphi_{0}^{2}}\right)^{-1 / 2},
$$

$$
r(x, y, t)=-\frac{\beta k^{2}}{k^{2}+\lambda^{2}}\left(\frac{3 \varphi_{0} \wp\left(\xi, g_{2}, g_{3}\right)+5 \delta \varphi_{0}-2 \gamma \varphi_{0}^{3}+3 K}{3 \wp\left(\xi, g_{2}, g_{3}\right)-\delta+2 \gamma \varphi_{0}^{2}}\right)^{-2} .
$$


(2) When $K<0, n=2, p=-\frac{1}{2}, \delta>0$ and $\gamma=0$, equation 1.2 has the following explicit bright solitary wave solutions:

$$
\begin{aligned}
& q(x, y, t)=\exp (i \theta)\left(\frac{-\delta}{K} \operatorname{sech}^{2}(\sqrt{\delta} \xi)\right)^{1 / 2} \\
& r(x, y, t)=-\frac{\beta k^{2}}{k^{2}+\lambda^{2}}\left(\frac{-\delta}{K} \operatorname{sech}^{2}(\sqrt{\delta} \xi)\right)^{2} .
\end{aligned}
$$

REMARK 3.2. In Figs. 1 and 2 we have plotted two- and three-dimensional surfaces for (3.31) and (3.33) for suitable values of parameters.

The solutions we obtained here are more abundant than those by means of the $\left(\frac{G^{\prime}}{G}\right)$ method [8], which only yields exact soliton solutions.

4. Conclusion. In this paper, the Weierstrass elliptic function method has been successfully applied to construct travelling wave solutions for the nonlinear differential equations such as nonlinear 2D Davey-Stewartson equation (DSE) with power law nonlinearity. The solutions obtained are solitary wave solutions, periodic wave solutions and bright solitary wave solutions. It has been observed that the traveling wave solutions of (3.44), (3.45), (3.46) and (3.47) obtained in this paper are new complex hyperbolic function solutions. The Weierstrass elliptic function method is effective in finding exact solutions of many other similar equations which have arbitrary-order nonlinearity.

\section{Appendix}

5.1. Elementary properties of Weierstrass functions. The original constructions of elliptic functions are due to Weierstrass [30] and Jacobi [20]. Here we list some elementary properties of Weierstrass functions $[2,6,24,31$. We define

$$
\begin{aligned}
\wp(z):= & \frac{1}{z^{2}} \\
& +\sum_{\{m, n\} \neq\{0,0\}}\left(\frac{1}{\left(z+2 m \omega_{1}+2 n \omega_{2}\right)^{2}}-\frac{1}{\left(2 m \omega_{1}+2 n \omega_{2}\right)^{2}}\right) .
\end{aligned}
$$

By construction, this function is doubly periodic with fundamental periods $2 \omega_{1}$ and $2 \omega_{2}$ :

$$
\wp\left(z+2 \omega_{1}\right)=\wp(z), \quad \wp\left(z+2 \omega_{2}\right)=\wp(z) .
$$

This function is called the Weierstrass elliptic function. 


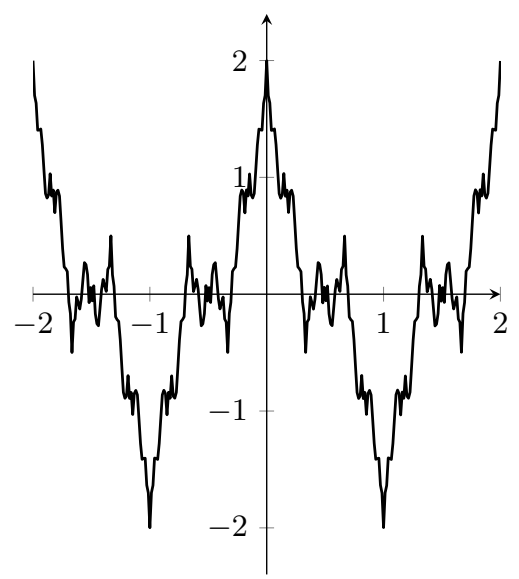

Basic properties. Directly from the definition (5.1), the Weierstrass elliptic function is even,

$$
\wp(-z)=\wp(z),
$$

and

$$
\wp(z)=\sum_{m, n} \frac{1}{\left(z+m \omega_{1}+n \omega_{2}\right)^{2}}-\sum_{\{m, n\} \neq\{0,0\}} \frac{1}{\left(m \omega_{1}+n \omega_{2}\right)^{2}} .
$$

Let us find the Laurent series of $\wp(z)$ at $z=0$. It is easy to show that

$$
\frac{1}{(z+w)^{2}}-\frac{1}{w^{2}}=\frac{1}{w^{2}} \sum_{k=1}^{\infty}(k+1)\left(-\frac{z}{w}\right)^{k} \text {. }
$$

Consequently,

$$
\wp(z)=\frac{1}{z^{2}}+\sum_{k=1}^{\infty} \sum_{\{m, n\} \neq\{0,0\}} \frac{(k+1)(-1)^{k}}{\left(m \omega_{1}+n \omega_{2}\right)^{k+2}} z^{k}=\frac{1}{z^{2}}+\sum_{k=1}^{\infty} a_{k} z^{k},
$$

where

$$
a_{k}=(k+1)(-1)^{k} \sum_{\{m, n\} \neq\{0,0\}} \frac{1}{\left(2 m \omega_{1}+2 n \omega_{2}\right)^{k+2}} .
$$

The fact that $\wp$ is even implies that only the even-indexed coefficients do not vanish,

$$
\begin{aligned}
a_{2 l+1} & =0, \\
a_{2 l} & =(2 l+1) \sum_{\{m, n\} \neq\{0,0\}} \frac{1}{\left(2 m \omega_{1}+2 n \omega_{2}\right)^{2(l+1)}} .
\end{aligned}
$$


The first terms are

$$
\begin{aligned}
& a_{2}=3 \sum_{\{m, n\} \neq\{0,0\}} \frac{1}{\left(2 m \omega_{1}+2 n \omega_{2}\right)^{4}}, \\
& a_{4}=5 \sum_{\{m, n\} \neq\{0,0\}} \frac{1}{\left(2 m \omega_{1}+2 n \omega_{2}\right)^{6}} .
\end{aligned}
$$

For reasons that will become apparent later, we define $g_{2}$ and $g_{3}$ so that

$$
\wp(z)=\frac{1}{z^{2}}+\frac{g_{2}}{20} z^{2}+\frac{g_{3}}{28} z^{4}+\mathcal{O}\left(z^{6}\right),
$$

implying that

$$
\begin{aligned}
& g_{2}=60 \sum_{\{m, n\} \neq\{0,0\}} \frac{1}{\left(2 m \omega_{1}+2 n \omega_{2}\right)^{4}}, \\
& g_{3}=140 \sum_{\{m, n\} \neq\{0,0\}} \frac{1}{\left(2 m \omega_{1}+2 n \omega_{2}\right)^{6}} .
\end{aligned}
$$

Weierstrass differential equation. By directly differentiating equation (5.1), the derivative of the Weierstrass function can be expressed as

$$
\wp^{\prime}(z)=-2 \sum_{m, n} \frac{1}{\left(z+m \omega_{1}+n \omega_{2}\right)^{3}} .
$$

It follows that this derivative is odd,

$$
\wp^{\prime}(-z)=-\wp^{\prime}(z) \text {. }
$$

The Laurent series of $\wp^{\prime}(z), \wp^{3}(z)$ and $\wp^{\prime 2}(z)$ at $z=0$ are

$$
\begin{array}{r}
\wp(z)=\frac{1}{z^{2}}+\frac{g_{2}}{20} z^{2}+\frac{g_{3}}{28} z^{4}+\mathcal{O}\left(z^{6}\right), \\
\wp^{\prime}(z)=-\frac{2}{z^{3}}+\frac{g_{2}}{10} z+\frac{g_{3}}{7} z^{3}+\mathcal{O}\left(z^{5}\right), \\
\wp^{3}(z)=\frac{1}{z^{6}}+\frac{3 g_{2}}{20} \frac{1}{z^{2}}+\frac{3 g_{3}}{28}+\mathcal{O}\left(z^{2}\right), \\
\wp^{\prime 2}(z)=\frac{4}{z^{6}}-\frac{2 g_{2}}{5} \frac{1}{z^{2}}-\frac{4 g_{3}}{7}+\mathcal{O}\left(z^{2}\right) .
\end{array}
$$

It is not difficult to show that there is a linear combination of the above which is not singular at $z=0$ and furthermore it vanishes there. One can eliminate the sixth order pole by taking an appropriate combination of $\wp^{\prime 2}$ and $\wp^{3}$. This leaves a function with a second order pole. Taking an appropriate combination of the latter combination and $\wp$ yields a function with no poles at $z=0$. Trivially, adding an appropriate constant results in a nonsingular function vanishing at $z=0$. The appropriate combination turns out to be

$$
\wp^{\prime 2}(z)-4 \wp^{3}(z)+g_{2} \wp(z)+g_{3}=\mathcal{O}\left(z^{2}\right) .
$$


But the derivative as well as powers of an elliptic function are elliptic functions with the same periods. Therefore, the function $\wp^{\prime 2}(z)=4 \wp^{3}(z)-$ $g_{2} \wp(z)-g_{3}$ is an elliptic function with the same periods as $\wp(z)$. Since the latter has no pole at $z=0$, it does not have any pole at all, and thus it is an elliptic function with no poles. As elliptic functions with no poles are necessarily constants and since $\wp^{\prime 2}(z)=4 \wp^{3}(z)-g_{2} \wp(z)-g_{3}$ vanishes at the origin, it vanishes everywhere. This implies that the Weierstrass elliptic function obeys the differential equation

$$
\wp^{\prime 2}(z)=4 \wp^{3}(z)-g_{2} \wp(z)-g_{3}=0 .
$$

This differential equation is of great importance in applications of the Weierstrass elliptic function in physics. For a physicist it is sometimes useful to even treat this differential equation as the definition of the Weierstrass elliptic function.

It turns out that the Weierstrass elliptic function is the general solution of the differential equation

$$
\left(\frac{d y}{d z}\right)^{2}=4 y^{3}-g_{2} y-g_{3} .
$$

After the substitution $y=\wp(w)$, equation (5.13) assumes the form

$$
\left(\frac{d w}{d z}\right)^{2}=1
$$

which obviously has the solutions $w= \pm z+z_{0}$. This implies that $y=$ $\wp\left( \pm z+z_{0}\right)$ and since the Weierstrass elliptic function is even, the general solution of the Weierstrass equation (5.13) can be written in the form

$$
y=\wp\left(z+z_{0}\right) .
$$

In the following, we will deduce an integral formula for the inverse function of $\wp$. In order to do so, we define

$$
z(y):=\int_{y}^{\infty} \frac{1}{\sqrt{4 t^{3}-g_{2} t-g_{3}}} d t .
$$

Differentiating with respect to $z$ one gets

$$
1=-\frac{d y}{d z} \frac{1}{\sqrt{4 y^{3}-g_{2} y-g_{3}}} \text { so }\left(\frac{d y}{d z}\right)^{2}=4 y^{3}-g_{2} y-g_{3} .
$$

We have just shown that the general solution of this equation is

$$
y=\wp\left(z+z_{0}\right) .
$$

Since the integral in 5.15 converges, it should vanish as $y \rightarrow \infty$, or equivalently $\lim _{y \rightarrow \infty} z(y)=0$. This implies that $z=z_{0}$ is the position of a pole, 
or in other words it is congruent to $z=0$. This means that

$$
y=\wp\left(z+2 m \omega_{1}+2 n \omega_{2}\right)=\wp(z) .
$$

Substituting the above into the equation (5.15) yields the integral formula for the Weierstrass elliptic function,

$$
z=\int_{\wp(z)}^{\infty} \frac{1}{\sqrt{4 t^{3}-g_{2} t-g_{3}}} d t .
$$

One could wonder how the above formula is consistent with the fact that $\wp$ is an elliptic function, and thus all numbers congruent to each other should be mapped to the same value of $\wp$. The answer to this question is that the integrable quantity in (5.16) has branch cuts. Depending on the selection of the path from $\wp(z)$ to infinity and more specifically depending on how many times the path encircles each branch cut, one may get any number congruent to $z$ or $-z$. A more precise expression of the integral formula is

$$
\int_{\wp(z)}^{\infty} \frac{1}{\sqrt{4 t^{3}-g_{2} t-g_{3}}} d t \sim \pm z .
$$

The roots of the cubic polynomial. We denote the values of the Weierstrass elliptic function at the half-periods $\omega_{1}, \omega_{2}$ and $\omega_{3}:=\omega_{1}+\omega_{2}$ as

$$
e_{1}:=\wp\left(\omega_{1}\right), \quad e_{2}:=\wp\left(\omega_{3}\right), \quad e_{3}:=\wp\left(\omega_{2}\right) .
$$

The permutation between the indices of $\omega$ 's and $e$ 's is introduced for notational reasons that will become apparent later. The periodicity properties of $\wp$ combined with the fact that the latter is an even function imply that $\wp$ is stationary at the half-periods. For example,

$$
\wp^{\prime}\left(\omega_{1}\right)=-\wp^{\prime}\left(-\omega_{1}\right)=-\wp^{\prime}\left(2 \omega_{1}-\omega_{1}\right)=-\wp^{\prime}\left(\omega_{1}\right),
$$

implying that $\wp^{\prime}\left(\omega_{1}\right)=0$. Similarly one can show that

$$
\wp^{\prime}\left(\omega_{1}\right)=\wp^{\prime}\left(\omega_{2}\right)=\wp^{\prime}\left(\omega_{3}\right)=0 .
$$

Substituting a half-period into the Weierstrass equation (5.13), we get

$$
4 e_{i}^{3}-g_{2} e_{i}-g_{3}=0 .
$$

The derivative of $\wp$, as shown in (5.10), has a single third order pole in each cell, congruent to $z=0$. Thus, $\wp^{\prime}$ is an elliptic function of order 3 and therefore it has exactly three roots in each cell. Since $\omega_{1}, \omega_{2}$ and $\omega_{3}$ all lie within the fundamental period parallelogram, they cannot be congruent to each other, and thus, there is no other root within the latter. This also implies that $\omega_{1}, \omega_{2}$ and $\omega_{3}$ are necessarily first order roots of $\wp^{\prime}$. All other roots of $\wp^{\prime}$ are congruent to those. Finally, when equation 5.20 has a double root, the solution of the differential equation (5.13) cannot be an elliptic function. 
An implication of the above is that $z=\omega_{1}, z=\omega_{2}$ and $z=\omega_{3}$ are the only locations within the fundamental period parallelogram, where the Laurent series of the function $\wp(z)-\wp\left(z_{0}\right)$ has a vanishing first order term in the region of $z_{0}$. Consequently, the equation $\wp(z)=f_{0}$ has a double root only when $f_{0}$ equals any of the three roots $e_{1}, e_{2}$ or $e_{3}$. Since $\wp$ is an order two elliptic function, the complex numbers $e_{1}, e_{2}$ and $e_{3}$ are the only ones appearing only once in a cell, whereas all other complex numbers appear twice.

Finally, equation 5.20 implies that $e_{i}$ are the three roots of the polynomial appearing on the right hand side of the Weierstrass equation, namely

$$
Q(t):=4 t^{3}-g_{2} t-g_{3}=4\left(t-e_{1}\right)\left(t-e_{2}\right)\left(t-e_{3}\right) .
$$

This directly implies that $e_{i}$ obey

$$
\begin{aligned}
e_{1}+e_{2}+e_{3} & =0, \\
e_{1} e_{2}+e_{2} e_{3}+e_{3} e_{1} & =-g_{2} / 4, \\
e_{1} e_{2} e_{3} & =g_{3} / 4 .
\end{aligned}
$$

Other properties. The Weierstrass elliptic function obeys the homogeneity relation

$$
\wp\left(z ; g_{2}, g_{3}\right)=\mu^{2} \wp\left(\mu z ; \frac{g_{2}}{\mu^{4}}, \frac{g_{3}}{\mu^{6}}\right) .
$$

For the specific case $\mu=i$, the above relation assumes the form

$$
\wp\left(z ; g_{2}, g_{3}\right)=-\wp\left(i z ; g_{2},-g_{3}\right) .
$$

Finally, when two of the roots $e_{1}, e_{2}$ and $e_{3}$ coincide, the Weierstrass elliptic function degenerates to a simply periodic function. Assuming that the moduli $g_{2}$ and $g_{3}$ are real, the existence of a double root implies that all roots are real. When the double root is larger than the simple root, the Weierstrass elliptic function takes the form

$$
\wp\left(z ; 12 e_{0}^{2},-8 e_{0}^{3}\right)=e_{0}+\frac{3 e_{0}}{\sinh ^{2}\left(\sqrt{3 e_{0}} z\right)},
$$

whereas when the double root is smaller than the simple root, it takes the form

$$
\wp\left(z ; 12 e_{0}^{2}, 8 e_{0}^{3}\right)=-e_{0}+\frac{3 e_{0}}{\sin ^{2}\left(\sqrt{3 e_{0}} z\right)} .
$$

If there is only one triple root, then it must be vanishing, since the three roots sum to zero. In this case, the Weierstrass elliptic function degenerates to a function that is not periodic at all, namely

$$
\wp(z ; 0,0)=1 / z^{2} .
$$




\section{References}

[1] M. Abramovitz and I. A. Stegun, Handbook of Mathematical Functions, 9th ed., Dover, New York, 1972.

[2] N. I. Akhiezer, Elements of the Theory of Elliptic Functions, Transl. Math. Monogr. 79, Amer. Math. Soc., Providence, RI, 1990.

[3] A. Bekirand A. C. Cevikel, New solitons and periodic solutions for nonlinear physical models in mathematical physics, Nonlinear Anal. Real World Appl. 11 (2010), 32753285.

[4] A. H. Bhrawy, M. A. Abdelkawy and A. Biswas, Cnoidal and snoidal wave solutions to coupled nonlinear wave equations by the extended Jacobi's elliptic function method, Comm. Nonlinear Sci. Numer. Simulation 18 (2013), 915-925.

[5] M. Boiti, J. J.-P. Leon, L. Martina and F. Pempinelli, Scattering of localized solitons in the plane, Phys. Lett. A 132 (1988), 432-439.

[6] K. Chanderasekharam, Elliptic Functions, Springer, Berlin, 1985.

[7] A. Davey and K. Stewartson, On three-dimensional packets of surfaces waves, Proc. Roy. Soc. London Ser. A 338 (1974), 101-110.

[8] G. Ebadi and A. Biswas, The $\frac{G^{\prime}}{G}$ method and 1-soliton solution of the Davey-Stewartson equation, Math. Computer Modelling 53 (2011), 694-698.

[9] A. El Achab, Constructing of exact solutions to the nonlinear Schrödinger equation (NLSE) with power-law nonlinearity by the Weierstrass elliptic function method, Optik 127 (2016), 1229-1232.

[10] A. El Achab, Elliptic travelling wave solutions to a generalized boussinesq equation, Abstr. Appl. Anal. 2014, art. 256019, 7 pp.

[11] A. El Achab, On the integrability of the generalized Pochhammer-Chree (PC) equations, Phys. A 545 (2020), art. 123576, 9 pp.

[12] A. El Achab, Weierstrass elliptic solutions to a Zakharov equation in plasmas with power law nonlinearity, Appl. Math. (Warsaw) 42 (2015), 13-22.

[13] A. El Achab and A. Amine, A construction of new exact periodic wave and solitary wave solutions for the 2D Ginzburg-Landau equation, Nonlinear Dynam. 91 (2018), 995-999.

[14] A. S. Fokas and P. M. Santini, Coherent structures in multidimensions, Phys. Rev. Lett. 63 (1989), 1329-1333.

[15] A. S. Fokas and P. M. Santini, Dromions and a boundary value problem for the Davey-Stewartson 1 equation, Phys. D 44 (1990), 99-130.

[16] Y. Gurefe, E. Misirli, Y. Pandir, A. Sonmezoglu and M. Ekici, New exact solutions of the Davey-Stewartson equation with power-law nonlinearity, Bull. Malays. Math. Sci. Soc. 38 (2015), 1223-1234.

[17] J. Hietarinta, One-dromion solutions for generic classes of equations, Phys. Lett. A 149 (1990), 113-118.

[18] J. Hietarinta and R. Hirota, Multidromion solutions to the Davey-Stewartson equation, Phys. Lett. A 145 (1990), 237-244.

[19] E. L. Ince, Ordinary Differential Equations, Dover, New York, 1956.

[20] C. G. J. Jacobi, Fundamenta Nova Theoriae Functionum Ellipticarum, Borntraeger, Königsberg, 1829.

[21] H. Jafari and M. Alipour, Solution of the Davey-Stewartson equation using homotopy analysis method, Nonlinear Anal. Model. Control 15 (2010), 423-433.

[22] H. Jafari, A. Sooraki, Y. Talebi and A. Biswas, The first integral method and traveling wave solutions to Davey-Stewartson equation, Nonlinear Anal. Model. Control 17 (2012), 182-193. 
[23] H. Leblond, Spatiotemporal optical pulse control using microwaves, Phys. Rev. Lett. 95 (2005), no. 3, art. 033902, 4 pp.

[24] A. Lesfari, Introduction à la géométrie algébrique complexe, Hermann, Paris, 2015.

[25] M. Mirzazadeh, Soliton solutions of Davey-Stewartson equation by trial equation method and ansatz approach, Nonlinear Dynam. 82 (2015), 1775-1780.

[26] J. Nickel, V. S. Serov and H. W. Schurmann, Some elliptic travelling wave solutions for Novikov-Veselov equation, Progr. Electromagnetc Res. 61 (2006), 323-331.

[27] R. Radha and M. Lakshmanan, Localized coherent structures and integrability in a generalized (2+1)-dimensional nonlinear Schrödinger equation, Chaos Solitons Fractals 8 (1997), 17-25.

[28] H. W. Schurmann, V. S. Serov and J. Nickel, Superposition in nonlinear wave and evolution equations, Int. J. Theoret. Phys. 45 (2006), 1057-1073.

[29] J. Shi, J. Li and S. Li, Analytical travelling wave solutions and parameter analysis for the (2+1)-dimensional Davey-Stewartson-type equations, Pramana-J. Phys. 81 (2013), 747-762.

[30] K. Weierstrass, Mathematische werke V, Johnson, New York, 1915.

[31] E. T. Whittaker and G. Watson, A Course of Modern Analysis, Cambridge Univ. Press, Cambridge, 1988.

[32] H. A. Zedan and S. J. Monaquel, The sine-cosine method for the Davey-Stewartson equations, Appl. Math. E-Notes 10 (2010), 103-111.

[33] H. A. Zedan and S. Sh. Tantawy, Solution of Davey-Stewartson equations by homotopy perturbation method, Comput. Math. Math. Phys. 49 (2009), 1382-1388.

\author{
Abdelfattah El Achab \\ LIBMA Laboratory \\ Department of Mathematics \\ Faculty of Sciences Semlalia \\ University Cadi Ayyad \\ Bd. du Prince Moulay Abdellah, B.P. 2390 \\ Marrakech 40.000, Morocco \\ E-mail: abdelfattahelachab@gmail.com
}

